\title{
L'ISTITUZIONE DI UNA «CATTEDRA» JEMOLO AD OXFORD
}

È stata istituita una «Cattedra» intestata ad Arturo Carlo Jemolo presso il Centro di Studi Europei del Nuffield College di Oxford, con il finanziamento della Banca d'Italia, della Banca Commerciale Italiana, del Banco di Santo Spirito, dell'Istituto Bancario S. Paolo di Torino e delle Assicurazioni Generali.

Lo scopo della «cattedra» è di consentire sia a ricercatori ed esperti italiani, sia a ricercatori ed esperti non italiani (che studiano problemi sociali e giuridici italiani) di compiere le loro ricerche presso il Centro, svolgendo in esso anche un'attività di insegnamento.

La «Cattedra» è destinata a studiosi ed esperti di economia, sociologia, scienze politiche, storia moderna e diritto pubblico.

$\mathrm{La}$ «Cattedra» è assegnata, previa selezione svolta da un apposito comitato, dal Nuffield College e comporta la residenza nel College per periodi oscillanti da un mese a due mesi, che possono estendersi anche a un periodo superiore, il pagamento dei costi di viaggio e di soggiorno, l'accesso in tutte le biblioteche e la disponibilità di una segreteria presso il Centro.

Le candidature possono essere inviate, con indicazione del programma di ricerca, del periodo, un breve curriculum vitae e i nomi di due «presentatori» a: The Warden, Nuffield College, Oxford OX1 1NF. 